\title{
Integration method of 3D MR spectroscopy into treatment planning system for glioblastoma IMRT dose painting with integrated simultaneous boost
}

Soléakhéna Ken ${ }^{1,2^{*}}$, Laure Vieillevigne ${ }^{1}$, Xavier Franceries ${ }^{2}$, Luc Simon ${ }^{1}$, Caroline Supper ${ }^{1}$, Jean-Albert Lotterie ${ }^{2,3}$, Thomas Filleron ${ }^{1}$, Vincent Lubrano ${ }^{2,3}$, Isabelle Berry ${ }^{2,3}$, Emmanuelle Cassol $^{2,3}$, Martine Delannes ${ }^{1}$, Pierre Celsis ${ }^{2}$, Elizabeth Moyal Cohen-Jonathan ${ }^{1,4}$ and Anne Laprie ${ }^{1,2}$

\begin{abstract}
Background: To integrate 3D MR spectroscopy imaging (MRSI) in the treatment planning system (TPS) for glioblastoma dose painting to guide simultaneous integrated boost (SIB) in intensity-modulated radiation therapy (IMRT).

Methods: For sixteen glioblastoma patients, we have simulated three types of dosimetry plans, one conventional plan of 60-Gy in 3D conformational radiotherapy (3D-CRT), one 60-Gy plan in IMRT and one 72-Gy plan in SIB-IMRT. All sixteen MRSI metabolic maps were integrated into TPS, using normalization with color-space conversion and threshold-based segmentation. The fusion between the metabolic maps and the planning CT scans were assessed. Dosimetry comparisons were performed between the different plans of 60-Gy 3D-CRT, 60-Gy IMRT and 72-Gy SIB-IMRT, the last plan was targeted on MRSI abnormalities and contrast enhancement (CE).

Results: Fusion assessment was performed for 160 transformations. It resulted in maximum differences $<1.00 \mathrm{~mm}$ for translation parameters and $\leq 1.15^{\circ}$ for rotation. Dosimetry plans of $72-$ Gy SIB-IMRT and 60-Gy IMRT showed a significantly decreased maximum dose to the brainstem (44.00 and 44.30 vs. $57.01 \mathrm{~Gy}$ ) and decreased high dose-volumes to normal brain (19 and 20 vs. $23 \%$ and 7 and 7 vs. 12\%) compared to 60-Gy 3D-CRT $(p<0.05)$.

Conclusions: Delivering standard doses to conventional target and higher doses to new target volumes characterized by MRSI and CE is now possible and does not increase dose to organs at risk. MRSI and CE abnormalities are now integrated for glioblastoma SIB-IMRT, concomitant with temozolomide, in an ongoing multi-institutional phase-III clinical trial. Our method of MR spectroscopy maps integration to TPS is robust and reliable; integration to neuronavigation systems with this method could also improve glioblastoma resection or guide biopsies.
\end{abstract}

Keywords: MR spectroscopy imaging (MRSI), Glioblastoma, Simultaneous integrated boost intensity modulation radiation therapy (SIB-IMRT),Dose painting

\footnotetext{
*Correspondence: Ken.Soleakhena@claudiusregaud.fr

'Department of Radiation Oncology and Medical Physics, Institut Claudius

Regaud, Toulouse 31052, France

${ }^{2}$ INSERM UMR 825 Imagerie cérébrale et handicaps neurologiques, Toulouse

31059, France

Full list of author information is available at the end of the article
} 


\section{Background}

Glioblastoma multiforme (GBM) is the most frequent malignant primary brain tumor in adult patients. Prognosis remains poor with a median survival of 14.6 months following treatment with surgery, external beam radiotherapy (RT), and chemotherapy [1]. Although adjuvant RT increases overall survival, whatever the age or Karnofsky/ OMS status of the patient, more than $90 \%$ of failures occur within the irradiated volumes [2]. This suggests that the dose conventionally delivered is not sufficient. Therefore, there is interest in increasing the dose to specific and more aggressive parts of the tumor while sparing normal tissue, using new technologies such as intensity-modulated radiation therapy (IMRT) [3,4].

As conventional MRI morphological sequences are insufficient to determine the potential target for a dose escalation [5] other types of imaging are needed, such as metabolic imaging [6,7]. The modality of proton magnetic resonance spectroscopy imaging (MRSI) is a relevant tool to define new targets as it can characterize the biochemical, metabolic and pathological changes in brain tissues [8-11] with the analysis of 3D-multi-voxel array within the MRI lesions and the surrounding normal tissue. MRSI data have been correlated with histopathology and can assess the residual disease after surgical resection in high-grade gliomas [12]. In addition, MRSI parameters were also found to be predictive of survival $[13,14]$.

The most common observation in glioblastoma is the peak corresponding to the choline-containing compounds (Cho) which increases with membrane proliferation, thus reflecting tumor presence and aggressiveness [15]. For relative quantification of MR spectroscopic data, the ratio of Cho over $\mathrm{N}$-acetyl-aspartate (NAA, a neurotransmitter only found in normal functioning neurons), is used [16]. The volumes corresponding to MRSI abnormalities and contrast enhancement (CE) were found to predict relapse patterns $[17,18]$, in concordance with our results obtained from a prospective trial [19]. MRSI (index of Cho/NAA $\geq 2$ ) could predict the extent of anatomical and metabolic relapse after radio-chemotherapy in patients with glioblastoma [20]. Therefore, these volumes represent potential radioresistant areas on which subvolume boosting [21] or dose painting by contours [22] is possible.

There are two main issues for the integration of MRSI into a RT treatment planning system (TPS). Firstly, MRSI images obtained from MRI scanners are MR spectroscopic maps overlaid on corresponding anatomical MR images. These images do not conform to DICOM standards, they are not compatible (contrarily to conventional MR images) for automatic image fusion with the planning CT scans. Secondly, the escalation in radiation dose from simultaneous integrated boost
(SIB) should be carefully evaluated, in particular for organs at risk (OAR).

We performed this study in order to prepare a multiinstitutional phase III prospective clinical trial of glioblastoma dose painting guided by MRSI. This trial will compare two RT treatments in concomitance with temozolomide: one delivering $60 \mathrm{~Gy}$ on conventional target volume and the other delivering 60 Gy on conventional target volume and a SIB of 72 Gy on a new target volume specific to MRSI.

In this paper, we propose an integration method of metabolic maps into TPS, overcoming the absence of DICOM 3.0 standard for MRSI, to guide the simultaneous integrated boost. We then compare dosimetry plans of standard 60-Gy treatment in 3D conformational radiotherapy (60-Gy 3D-CRT), 60-Gy in IMRT and the treatment with the dose escalation of 72 Gy in SIB-IMRT. The method that we described in this article can be used for future prospective trials integrating MR spectroscopy in radiotherapy planning treatments.

\section{Methods \\ Patients}

The pre-RT data were from 16 patients enrolled in a prospective clinical trial on farnesyl-transferase inhibitors (FTI) [19] associated with radiotherapy to treat glioblastoma. The trial was approved by the local ethics committee and patients provided their written informed consent. They received FTI and standard 3D-CRT. We prospectively performed MRSI acquisition before radiotherapy on this homogeneous group at the same session time that the classical MRI sequences.

\section{Data acquisition}

For all 16 GMB patients, MR imaging was performed on a 1.5 T Magnetom Avanto Siemens scanner (Erlangen, Germany). Pre- and post- gadolinium injected $(2 \mathrm{~mL} / \mathrm{kg}$ body weight) T1-weighted (T1-Gd) and Turbo-Spin-Echo T2-weighted (T2) images were acquired for anatomic MR evaluation with voxel resolution of $0.90 \times 0.90 \times 3.00 \mathrm{~mm}^{3}$.

3D-chemical-shift imaging (3D-CSI) for MRSI acquisition consisted of three phase-encoded gradients prior to read-out, resulting in a scan time of $8 \mathrm{~min}$. MRSI acquisition consisted of a Spin-Echo-based sequence with the following parameters: TR/TE $=1500 \mathrm{~ms} / 135 \mathrm{~ms}$ for lactate detection, and four excitations, $\mathrm{FOV}$ was set at $100 \times 100 \mathrm{~mm}^{2}$ for a CSI matrix of $16 \times 16$, with eight slices of $25.0 \mathrm{~mm}$ thickness, resulting in voxel resolution of $6.25 \times 6.25 \times 25.0 \mathrm{~mm}^{3}$, i.e. $1 \mathrm{~cm}^{3}$. The 3D-CSI box was positioned to cover the majority of abnormalities and normal appearing tissue, while avoiding regions that could corrupt the spectra - such as bone and subcutaneous lipids. Saturation bands were also positioned around the volume of interest (VOI) to suppress signals from 
excited regions outside the VOI, and to provide good in vivo fat suppression.

CT simulation images for RT planning of all 16 GBM patients were acquired in helical mode with voxel resolution of $0.98 \times 0.98 \times 2.50 \mathrm{~mm}^{3}$.

\section{Data processing}

The spectroscopic processing protocol consisted of water substraction, low-pass filtering, frequency shift correction, baseline correction, phase correction and curve fitting in the frequency domain. These steps of spectra processing were performed with the Siemens Syngo MR B17 Spectroscopy application (Erlangen, Germany).

\section{Consistency analysis}

After image processing, additional information specific to MRSI abnormalities was embedded in the normalized and segmented anatomic-metabolic images. Accuracy of automated fusion between CT and anatomic MR images is given to be submillimeter and subdegree with Syntegra toolbox (Pinnacle software version $8.0 \mathrm{~m}$, Philips Medical Systems, Milpitas, CA) [23]. We found it relevant to check all 16 patients' data sets to determine if normalization and threshold-based segmentation could wrongly influence the fusion process between $\mathrm{CT}$ scans and anatomic-metabolic images. Reliability and repeatability of the fusion were assessed for each patient's data with 10 successive coregistration transformations. The result of the fusion process was visually validated. For consistency analysis, the means of standard deviations (SD) and the means of maximum differences between translation and rotation parameters, along the $x$ (left-right), $y$ (anterior-posterior), and $z$ (head-feet) axes were computed.

\section{Dose-plan comparisons: 60-Gy 3D-C RT, 60-Gy IMRT and 72-Gy SIB-IMRT}

For the treatment plans delivering 60 Gy, i.e. 60-Gy 3D-CRT and 60-Gy IMRT, the gross target volume (GTV1) was defined as the anatomical contrastenhancing tumor visible on the T1-Gd images. The clinical target volume (CTV1), representing the subclinical tumor involvement, was defined as GTV1 + 17.0-mm expansion including the edema visible on the T2-weighted images. The planning target volume (PTV1) was defined as CTV1+3.0-mm margin. The dose calculation was performed according to the conventional prescription of 60 Gy delivered in fractions of 2 Gy for the PTV1.

For the 72-Gy SIB-IMRT treatment plan, the GTV2 was defined as the MRSI abnormalities (Cho/NAA $\geq$ 2.00). The CTV2 was defined as the GTV2 + 7.0- mm expansion including the contrast-enhancing tumor visible on the T1-Gd images. The PTV2 was defined as the CTV2 + 3.0-mm margin. The dose prescription was the following: 60 Gy on the PTV1 as defined above and
72 Gy on the PTV2 (SIB) delivered in fractions of $2.4 \mathrm{~Gy}$.

We wanted to use the radiobiological advantages of an integrated boost,and therefore, taking into account the alpha/beta $=3$ as calculated with the LQ model and the dose equivalent for tumor repopulation, $80 \mathrm{~Gy}$ as the $2 \mathrm{~Gy}$ per day are equivalent to 30 fractions of 2.4 Gy [24].

The TPS used in this study was Pinnacle version $8.0 \mathrm{~m}$ (Philips Medical Systems, Milpitas, CA). The dose was calculated with the collapsed cone convolution-superposition model. For SIB-IMRT, we used the Direct Machine Parameter Optimization module, which directly optimizes the number of monitor units and the multileaf collimator leaves.

For comparison with the treatment plans delivering 60 Gy (60-Gy 3D-CRT and 60-Gy IMRT), six different beam configurations of 72-Gy SIB-IMRT, for all 16 patients' data sets, were tested: 96 dose-plans were then simulated. The six different beam configurations consisted of the following: configuration $\mathrm{A}=3$ coplanar beams, configuration $\mathrm{B}=3$ coplanar beams with different angles from configuration $\mathrm{A}$, configuration $\mathrm{C}=5$ coplanar beams, configuration $\mathrm{D}=7$ coplanar beams, configuration $\mathrm{E}=9$ coplanar beams and configuration $\mathrm{F}=5$ non-coplanar beams ( 3 coplanar and 2 non-coplanar beams).

The treatment plans delivering 60 Gy (60-Gy 3D-CRT and 60-Gy IMRT) and the 72-Gy SIB-IMRT plans were compared using the following criteria:

- Target coverage $\left(I_{1}\right)$, conformity index $\left(I_{2}\right)$, and conformation number $(C N)$ for quantifying the degree of conformity [25]:

$$
\begin{aligned}
I_{1}= & P T V_{95 \%} / V_{T O T \_P T V} \\
I_{2}= & P T V_{95 \%} / V_{I S O \_} 95 \\
& P T V_{95 \%}=\text { Target volume }(\mathrm{PTV}) \text { receiving } \\
& 95 \% \text { of the prescription dose } \\
& V_{T O T \_P T V}=\text { Total volume of target }(\mathrm{PTV}) \\
& V_{I S O \_95}=\text { Isodose volume enclosed by } 95 \% \\
\text { of the prescription dose } & \\
C N= & I_{1} \times I_{2} \quad
\end{aligned}
$$

- Doses received by OAR: maximum dose at $1 \%$ of the optic chiasm and brainstem.

- The dose-volumes of interest for the normal brain were 18, 36, and 50 Gy given relative to the volume of normal brain (V18, V36 and V50).

\section{Statistical analysis}

Continuous variables were described as their medians and range. A Wilcoxon test compared the paired data. All $p$-values were two-sided, and for all statistical tests, differences were considered significant at the 5\% level. Stata (StataCorp LP, Texas, USA) was used for all statistical analyses. 


\section{Results}

\section{MRSI integration into TPS}

MRSI acquisition from all patients resulted in 3652 voxels $($ mean $=228.3, \mathrm{SD}=55.6)$, The $\mathrm{Cho} / \mathrm{NAA}$ ratio was thoroughly reviewed on a voxel-by-voxel basis: 3120 voxels were considered for the computation of metabolite Cho/NAA maps. Both MR images (Figure 1, first row) and 3D-MRSI were acquired in the same plane in order for co-registration to occur, and the resulting snapshots consisted of anatomic-metabolic images coded under the red-green-blue (RGB) colorspace with a DICOM extension format file, but these were not compatible for integration into TPS (Figure 1, third row).

These anatomic-metabolic images were processed with scripts written in Matlab ${ }^{\circledR}$ (MathWorks, Inc.,
Natick, USA) for integration into TPS. For the imageprocessing normalization step, the separation of anatomical MR images from the color metabolite ratio maps was made. Conversion of the metabolite ratio maps from RGB to hue-saturation-value (HSV) color-space [26] was performed to retrieve a single quantitative value (hue) from the metabolic map, which was proportional to Cho/NAA. The Siemens Syngo MR B17 Spectroscopy application (Erlangen, Germany) provided the local maximum of Cho/NAA for each CSI slice (i.e. the red color on the corresponding color table), these values were used as inputs to compute global normalization across the entire 3D-MRSI volume of acquisition. The same principle of color-space conversion is possible for other color tables from different MR spectroscopy posttreatment softwares.
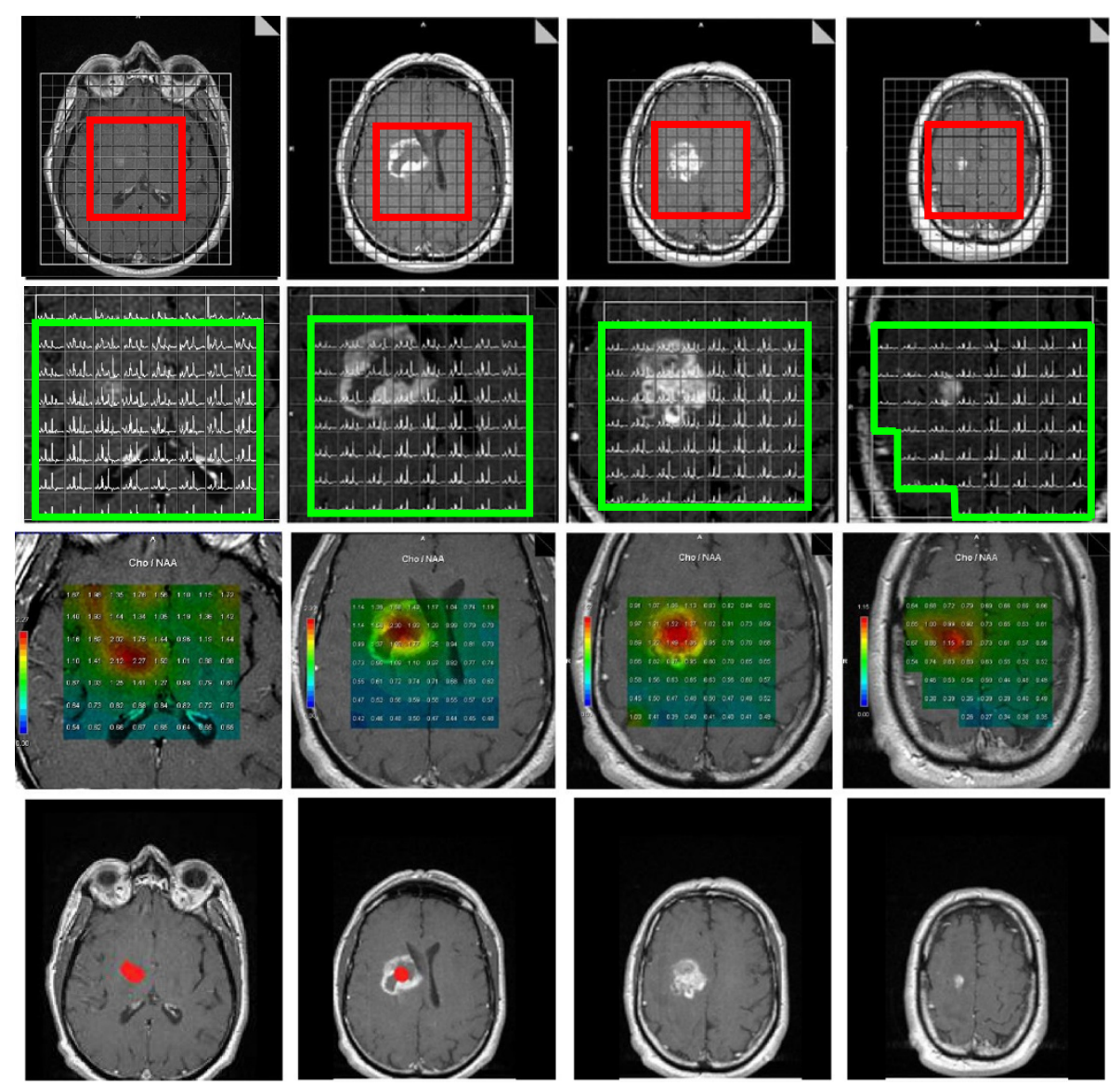

Figure 1 3D-MRSI acquisition before radiation therapy treatment of a 53 year-old unresected patient with confirmed glioblastoma located in the right capsulo-thalamic region (first row, the volume of acquisition is framed in red). On the T1-Gd anatomic MR images showing contrast-enhancing disease, the MRSI volume of interest is defined on a voxel by voxel basis,when alteration of metabolites spectra is observed, the voxel is rejected (green frame on second row). The anatomic-metabolic maps are computed from the above defined volume of interest (third row), the maximum Cho/NAA ratio values are encoded in red color and are respectively from left to right 2.27, 2.30, 1.52 and 1.15. The first two metabolite maps which present ratios of Cho/NAA $\geq 2.00$ suggest metabolic tumor activity. Regions of interest corresponding to ratio of Cho/NAA $\geq 2.00$ are obtained after normalization and threshold based segmentation from the anatomic-metabolic images, these ROls are highlighted in red (last row). Note on the first image (last row) that the location of the abnormal spectroscopic region is different and below the contrast-enhancing area. 
To extract the information specific to abnormal MRSI regions, the previously normalized maps were segmented with a threshold value of $\mathrm{Cho} / \mathrm{NAA} \geq 2.00$ [20]. Such segmented regions of interest, with abnormal metabolite index ratios, were then re-mapped onto respective anatomical MR images (Figure 1, last row) with smooth linear interpolation to the final resolution of anatomical MR images. In this study, the 3D-MRSI volume represented $24.84 \%$ and $13.63 \%$ of the T1-Gd and hyper-T2 volumes, respectively (range: $2.22-54.85 \%$ ). The integration was finalized with a copy of the DICOM headers from the T1-Gd images into the headers of the anatomic-metabolic images. These normalized and segmented anatomic-metabolic images were generated for all patients, and were successfully imported for fusion with planning CT scans under different TPS and evaluation software for RT (Pinnacle v8.0 m, Eclipse v8.9 and Artiview v2.6).

To summarize, the flow chart of image-processing steps that integrated MRSI-defined regions with abnormal Cho/ NAA ratio values into RT TPS is detailed in Figure 2.

\section{Consistency analysis of MRSI integration into TPS}

Results for the reliability and repeatability of the image fusion (10 successive co-registration transformations on each patient's data) were the following: means SD were found to be $0.19 \mathrm{~mm}, 0.25 \mathrm{~mm}, 0.39 \mathrm{~mm}, 0.25^{\circ}, 0.37^{\circ}$, and $0.29^{\circ}$ and means of maximum differences were found to be $0.59 \mathrm{~mm}, 0.49 \mathrm{~mm}, 0.83 \mathrm{~mm}, 0.82^{\circ}, 1.15^{\circ}$, and $0.94^{\circ}$,respectively for each translation and each rotation parameter, along the $x$ (left-right), $y$ (anterior-posterior), and $z$ (head-feet) axes.

\section{Dose-plan comparisons between 60-Gy 3D-CRT, 60-Gy IMRT and 72-Gy SIB-IMRT}

96 SIB-IMRT treatment plans were simulated and compared with 16 plans of 60-Gy IMRT and 16 standard 3D-CRT plans (Figure 3). Median volumes of PTV1 and PTV2 were respectively $307.76 \mathrm{~cm}^{3}$ (range: $84.52-$ $586.96 \mathrm{~cm}^{3}$ ) and $97.63 \mathrm{~cm}^{3}$ (range: $34.32-231.17 \mathrm{~cm}^{3}$ ).

Considering the PTV2 of the SIB-IMRT treatment plans, $I_{1}, I_{2}$, and $C N$ were evaluated and configuration

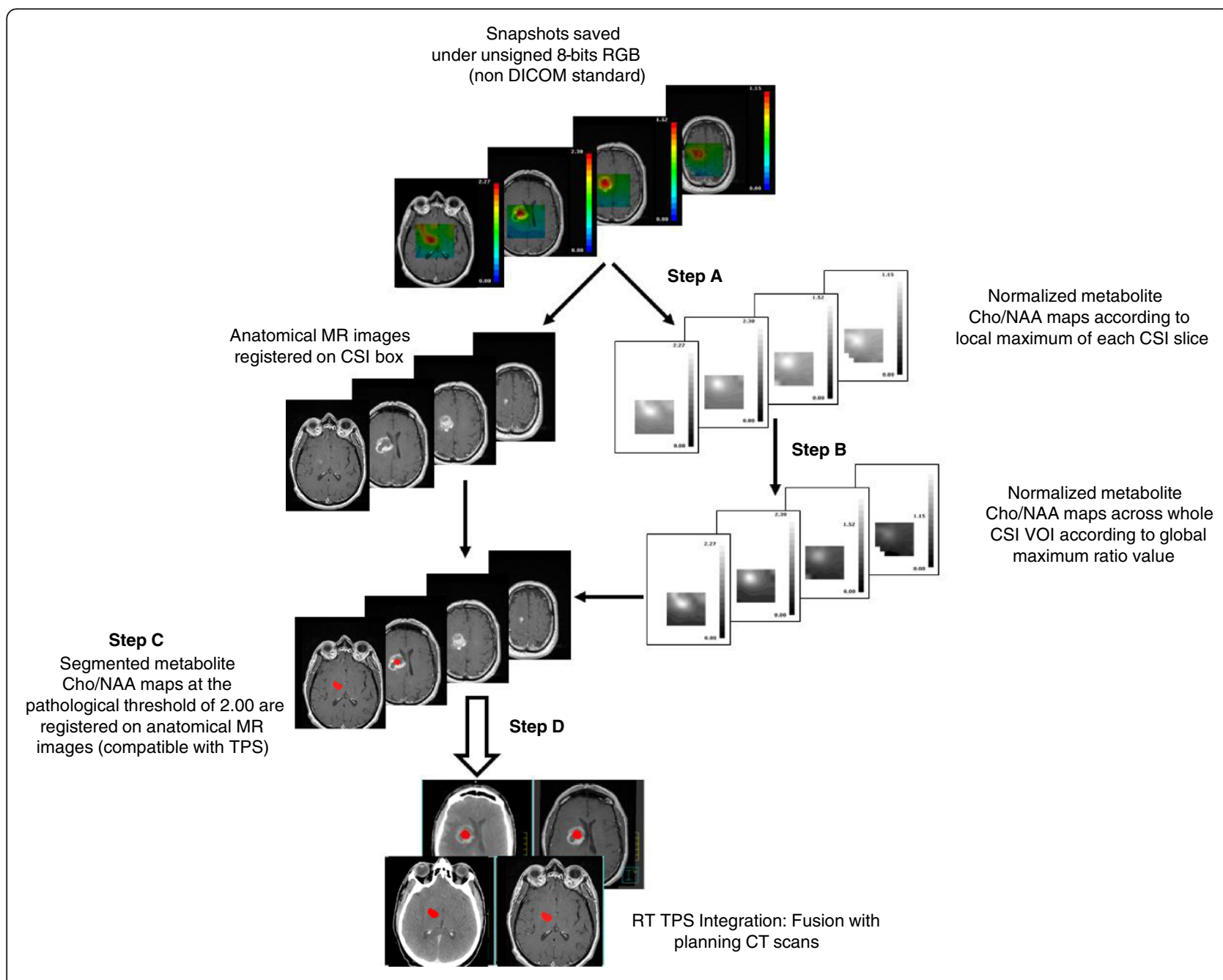

Figure 2 Flow chart of the image processing steps to integrate MRSI-defined regions with abnormal Cho/NAA ratio values into RT TPS. 
(a)

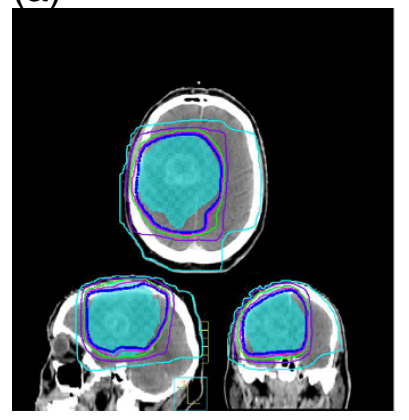

(b)

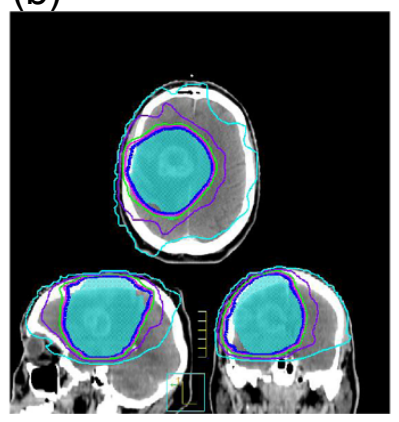

(c)

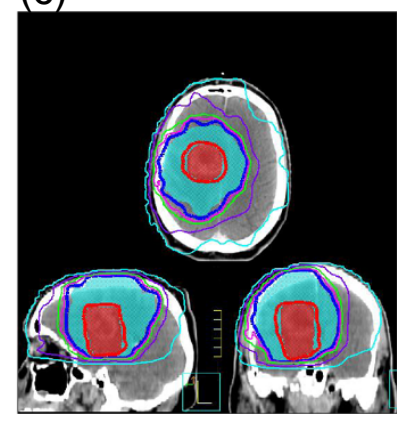

Figure 3 Comparison of dose plans between 60-Gy 3D-CRT, 60-Gy IMRT and 72-Gy SIB-IMRT. 60-Gy 3D-CRT and 60-Gy IMRT plans (respectively Figures $\mathbf{3} \mathbf{a}$ and $\mathbf{3 b}$ ) have one PTV1 color-washed in blue. The integration of Cho/NAA abnormal volumes defines new target relative to MRSI, i.e. PTV2 color-washed in red (Figure 3c), PTV1 is the same. The isodoses of 68.4 Gy (thick red isodose) and 57 Gy (thick dark blue isodose) represent 95\% of the prescribed dose respectively 72 Gy and 60 Gy on the PVT2 and PTV1. The isodose volumes of 54 Gy (pink), 50 Gy (green), 36 Gy (purple) and 18 Gy (light blue) for organs at risk sparing are also plotted.

C was not statistically different from configurations A, $\mathrm{D}, \mathrm{E}$, and $\mathrm{F}$, but higher $I_{1}, I_{2}$, and $C N$ were found compared with the configuration $B$ (respectively, 0.97 vs $0.95, p=0.005,0.79$ vs $0.74, p=0.030$ and 0.75 vs 0.68 , $p=0.034)$. Configuration $\mathrm{C}$ was then chosen for doseplan comparison with standard 60-Gy 3D-CRT and 60Gy IMRT with 5 beam configuration.

Considering the PTV1, the configuration $C$ shows no significant difference with configurations $\mathrm{A}, \mathrm{D}, \mathrm{E}$ and $\mathrm{F}$ but higher $I_{1}, I_{2}$ and $C N$ are found compared with the configuration $\mathrm{B}$ (respectively, 0.97 vs $0.95, p=0.005,0.88$ vs $0.84, p=0.013$ and 0.85 vs $0.82, p=0.001) . I_{1}, I_{2}$ and $C N$ for PTV1 are compared between 60-Gy 3D-CRT, 60-Gy IMRT and 72-Gy SIB-IMRT (configuration C). There is no significant difference for $I_{1}(0.98,0.95$ vs 0.97, $p>0.255$ ) but 60-Gy IMRT and 72-Gy SIB-IMRT plans performed significantly better for $I_{2}$ and $C N$ (respectively, $0.91,0.88$ vs $0.75, p<0.010$ and $0.84,0.85$ vs $0.72, p<0.035)$.

When comparing the maximum dose received by OAR, there was no statistically significant difference between 60-Gy 3D-CRT, 60-Gy IMRT and 72-Gy SIBIMRT for the optic chiasm $(p>0.088$, Figure 4a). Compared to 60 Gy 3D-CRT, 60-Gy IMRT and 72-Gy SIBIMRT significantly lowered the dose to the brainstem $(p<0.001$, Figure 4b).

For doses relative to the normal brain (Figure 4c), the dose-volume V18 was not significantly different, but V36 and V50 were significantly lower with 60-Gy IMRT and 72 - Gy SIB-IMRT $(p<0.05$ and $p<0.001)$.

\section{Discussion}

In this paper, we described an integration method of MRSI for radiotherapy treatment planning in order to perform a clinical trial for glioblastoma dose painting.
At a time when delivering a higher dose to such radioresistant tumors is possible with IMRT, defining the optimal target is of paramount importance.

Several studies with additional boost showed good tolerance for dose escalation [27] and sometimes improved tumor control $[3,28-30]$ but they were not systematically in concomitance with temozolomide and did not perform selective simultaneous integrated boost according to functional or metabolic imaging modalities; to our knowledge the only comparable approach was published by Piroth et al. [27], who described a prospective phase II study that defined the dose escalation (total dose of 72 Gy) with an integrated boost on active tumor as characterized by positron emission tomography (PET) using O-(2- $\left[{ }^{18} \mathrm{~F}\right]$ fluoroethyl)-L-tyrosine (FET), for which the results of survival data were comparable to standard treatment [1].

Despite the information contained in MRSI for predicting the site of relapse after radiotherapy $[17,20]$, 3D-MRSI still remains a challenging modality to integrate into TPS. Several attempts have been performed in the last decade [31,32], also in the radiosurgery field [33], but the innovative aspects of the MR spectroscopy integration presented here are the following: a) it is a method for three-dimensional MR spectroscopy that provides global normalization and threshold-based segmentation of the whole 3D-CSI volume of interest,b) it integrates metabolite ratio maps into TPS using co-registration with anatomic MR images, and c) it results in MR spectroscopic pre-defined regions ready to be contoured.

In this study, a consistency analysis was performed to assess the impact of combining MR anatomic and metabolic information on fusion with CT scans, as both MR and MRSI modalities were gathered in the same set of images.

As reliable integration of valuable biological target volumes specific to MRSI into TPS was reached, a 


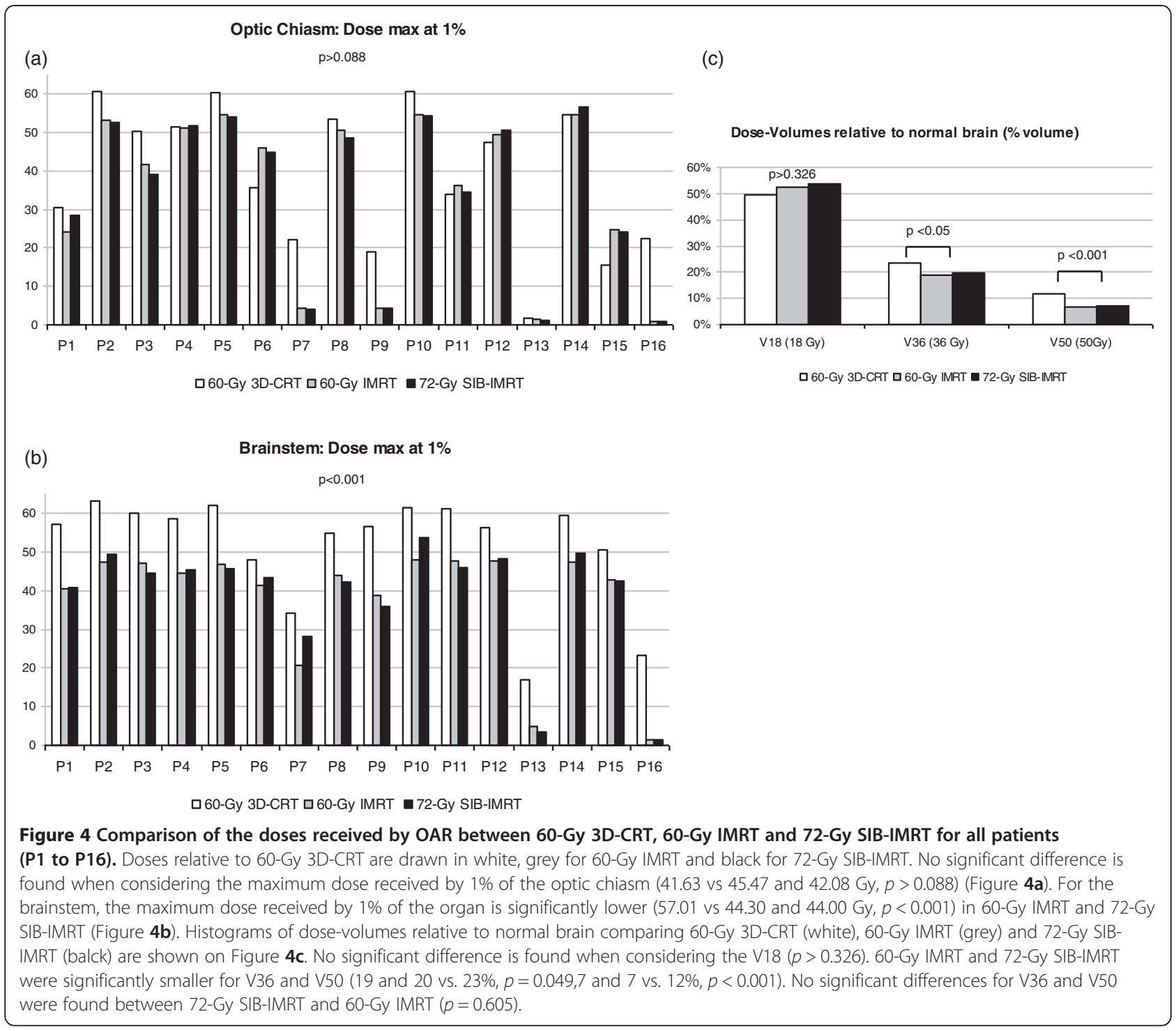

dosimetry study was performed to evaluate the impact on the OAR of dose escalation. SIB-IMRT allowed us to perform dose painting by contours for optimal irradiation of target volumes and optimal sparing of OAR [34]. Protection of normal brain tissue is of particular importance for tolerance to dose increases and to prevent radiation necrosis and neurocognitive deficits, as these are significantly correlated with the dose received by the normal brain [35]. Thus, a difficult compromise between radiation necrosis, neurocognitive impairment and tumor control has to be achieved. Although the dose is increased on the target volume, the dose received by OAR is either equivalent (optical chiasm) or significantly lowered with the MRSI guided 72-Gy SIB-IMRT compared to 60-Gy 3D-CRT (brainstem and normal brain) thanks to the IMRT technique. The repercussions of normal brain irradiation for patients with GBM will be more of an issue if patient survival is extended by dose escalation to regions that have a high risk of relapse.

\section{Conclusions}

We describe a reliable method to integrate 3D-MRSI for dose escalation on regions of high-risk of relapse while optimizing OAR sparing. This work represents a novel approach to the treatment of glioblastoma and is the basis of a multi-institutional phase-III prospective clinical trial, which is currently underway to compare conventional treatment delivering 60 -Gy versus 72-Gy SIB-IMRT guided by MRSI. This method could also be the basis of other innovative trials integrating MRSI in radiotherapy treatment planning but also in neuronavigation system to improve the GBM resection or guide biopsies. 


\section{Competing interests}

The authors declare that they have no competing interests.

\section{Authors' contributions}

$A L, E M C J$ and SK participated in the design and coordination of the study. $L V, C S$ and LS participated in the dose-plan comparison analysis. TF contributed to the statistical analysis. LV, CS, LS, AL and SK contributed to the interpretation of the data. SK and AL drafted the article. EMCJ, XF, JAL, $V L, I B, E C, M D$ and $P C$ critically reviewed/revised the article. All authors read and approved the final manuscript.

\section{Acknowledgements}

This work was part of the preparation of a multi-institutional trial, financed by the French National Institute for Cancer (INCa), which is comparing standard 3D-CRT delivering 60 Gy versus SIB-IMRT delivering 72 Gy guided by MR spectroscopy and contrast enhancement.

\section{Author details}

'Department of Radiation Oncology and Medical Physics, Institut Claudius Regaud, Toulouse 31052, France. ${ }^{2}$ INSERM UMR 825 Imagerie cérébrale et handicaps neurologiques, Toulouse 31059 , France. ${ }^{3}$ Centre Hospitalier Universitaire de Rangueil, Université Toulouse III Paul Sabatier, Toulouse 31059, France. ${ }^{4}$ INSERM UMR1037, CRCT, Institut Claudius Regaud, Toulouse 31052, France.

Received: 21 August 2012 Accepted: 27 December 2012 Published: 2 January 2013

\section{References}

1. Stupp R, Mason WP, van den Bent MJ, Weller M, Fisher B, Taphoorn MJ, Belanger K, Brandes AA, Marosi C, Bogdahn U, Curschmann J, Janzer RC, Ludwin SK, Gorlia T, Allgeier A, Lacombe D, Cairncross JG, Eisenhauer E, Mirimanoff RO: Radiotherapy plus concomitant and adjuvant temozolomide for glioblastoma. N Engl J Med 2005, 352:987-996.

2. Minniti G, Amelio D, Amichetti M, Salvati M, Muni R, Bozzao A, Lanzetta G, Scarpino S, Arcella A, Enrici RM: Patterns of failure and comparison of different target volume delineations in patients with glioblastoma treated with conformal radiotherapy plus concomitant and adjuvant temozolomide. Radiother Oncol 2010, 97:377-381.

3. Monjazeb AM, Ayala D, Jensen C, Case LD, Bourland JD, Ellis TL, McMullen KP, Chan MD, Tatter SB, Lesser GJ, Shaw EG: A phase I dose escalation study of hypofractionated IMRT field-in-field boost for newly diagnosed glioblastoma multiforme. Int J Radiat Oncol Biol Phys 2012, 82:743-748.

4. Amelio D, Lorentini S, Schwarz M, Amichetti M: Intensity-modulated radiation therapy in newly diagnosed glioblastoma: a systematic review on clinical and technical issues. Radiother Oncol 2010, 97:361-369.

5. Chan JL, Lee SW, Fraass BA, Normolle DP, Greenberg HS, Junck LR, Gebarski SS, Sandler HM: Survival and failure patterns of high-grade gliomas after three-dimensional conformal radiotherapy. J Clin Oncol 2002, 20:1635-1642.

6. Kallen K, Burtscher IM, Holtas S, Ryding E, Rosen I: 201Thallium SPECT and $1 \mathrm{H}-\mathrm{MRS}$ compared with MRI in chemotherapy monitoring of high-grade malignant astrocytomas. J Neurooncol 2000, 46:173-185.

7. Tsien Cl, Brown D, Normolle D, Schipper M, Morand P, Junck L, Heth JA, Gomez-Hassan D, Ten Haken R, Chenevert TL, Cao Y, Lawrence TS: Concurrent temozolomide and dose-escalated intensity modulated radiation therapy in newly diagnosed glioblastoma. Clin Cancer Res 2012, 18:273-279.

8. Alger JR, Frank JA, Bizzi A, Fulham MJ, DeSouza BX, Duhaney MO, Inscoe SW, Black JL, van Zijl PC, Moonen CT: Metabolism of human gliomas: assessment with $\mathrm{H}-1$ MR spectroscopy and F-18 fluorodeoxyglucose PET. Radiology 1990, 177:633-641.

9. Dowling C, Bollen AW, Noworolski SM, McDermott MW, Barbaro NM, Day MR, Henry RG, Chang SM, Dillon WP, Nelson SJ, Vigneron DB: Preoperative proton MR spectroscopic imaging of brain tumors: correlation with histopathologic analysis of resection specimens. AJNR Am J Neuroradiol 2001, 22:604-612.

10. Tzika AA: Proton magnetic resonance spectroscopic imaging as a cancer biomarker for pediatric brain tumors (review). Int J Oncol 2008, 32:517-526
11. Astrakas LG, Zurakowski D, Tzika AA, Zarifi MK, Anthony DC, De Girolami U, Tarbell NJ, Black PM: Noninvasive magnetic resonance spectroscopic imaging biomarkers to predict the clinical grade of pediatric brain tumors. Clin Cancer Res 2004, 10:8220-8228.

12. Pirzkall A, Li X, Oh J, Chang S, Berger MS, Larson DA, Verhey LJ, Dillon WP, Nelson SJ: 3D MRSI for resected high-grade gliomas before RT: tumor extent according to metabolic activity in relation to MRI. Int J Radiat Oncol Biol Phys 2004, 59:126-137.

13. Crawford FW, Khayal IS, McGue C, Saraswathy S, Pirzkall A, Cha S, Lamborn KR, Chang SM, Berger MS, Nelson SJ: Relationship of Pre-surgery metabolic and physiological MR imaging parameters to survival for patients with untreated GBM. J Neurooncol 2009, 91:337-351.

14. Saraswathy S, Crawford FW, Lamborn KR, Pirzkall A, Chang S, Cha S, Nelson $\mathrm{SJ}$ : Evaluation of MR markers that predict survival in patients with newly diagnosed GBM prior to adjuvant therapy. J Neurooncol 2009, 91:69-81.

15. Sankar T, Caramanos Z, Assina R, Villemure JG, Leblanc R, Langleben A, Arnold DL, Preul MC: Prospective serial proton MR spectroscopic assessment of response to tamoxifen for recurrent malignant glioma. J Neurooncol 2008, 90:63-76.

16. McKnight TR, dem Bussche MH, Vigneron DB, Lu Y, Berger MS, McDermott MW, Dillon WP, Graves EE, Pirzkall A, Nelson SJ: Histopathological validation of a three-dimensional magnetic resonance spectroscopy index as a predictor of tumor presence. J Neurosurg 2002, 97:794-802.

17. Park I, Tamai G, Lee MC, Chuang CF, Chang SM, Berger MS, Nelson SJ, Pirzkall A: Patterns of recurrence analysis in newly diagnosed glioblastoma multiforme after three-dimensional conformal radiation therapy with respect to Pre-radiation therapy magnetic resonance spectroscopic findings. Int J Radiat Oncol Biol Phys 2007, 69:381-389.

18. Zeng QS, Li CF, Liu H, Zhen JH, Feng DC: Distinction between recurrent glioma and radiation injury using magnetic resonance spectroscopy in combination with diffusion-weighted imaging. Int J Radiat Oncol Biol Phys 2007, 68:151-158.

19. Moyal EC, Laprie A, Delannes M, Poublanc M, Catalaa I, Dalenc F, Berchery D, Sabatier J, Bousquet P, De Porre P, Alaux B, Toulas C: Phase I trial of tipifarnib (R115777) concurrent with radiotherapy in patients with glioblastoma multiforme. Int J Radiat Oncol Biol Phys 2007, 68:1396-1401.

20. Laprie A, Catalaa I, Cassol E, McKnight TR, Berchery D, Marre D, Bachaud JM, Berry I, Moyal EC: Proton magnetic resonance spectroscopic imaging in newly diagnosed glioblastoma: predictive value for the site of postradiotherapy relapse in a prospective longitudinal study. Int J Radiat Oncol Biol Phys 2008, 70:773-781.

21. Bentzen SM, Gregoire V: Molecular imaging-based dose painting: a novel paradigm for radiation therapy prescription. Semin Radiat Oncol 2011, 21:101-110.

22. Meijer G, Steenhuijsen J, Bal M, De Jaeger K, Schuring D, Theuws J: Dose painting by contours versus dose painting by numbers for stage II/III lung cancer: practical implications of using a broad or sharp brush. Radiother Oncol 2011, 100:396-401.

23. Zhang Y, Chu JC, Hsi W, Khan AJ, Mehta PS, Bernard DB, Abrams RA: Evaluation of four volume-based image registration algorithms. Med Dosim 2009, 34:317-322.

24. Jones B, Sanghera P: Estimation of radiobiologic parameters and equivalent radiation dose of cytotoxic chemotherapy in malignant glioma. Int J Radiat Oncol Biol Phys 2007, 68:441-448.

25. Lomax NJ, Scheib SG: Quantifying the degree of conformity in radiosurgery treatment planning. Int J Radiat Oncol Biol Phys 2003, 55:1409-1419.

26. Smith AR: Color gamut tranform pairs. SIGGRAPH 78 Conference Proceedings 1978, 12:12-19.

27. Piroth MD, Pinkawa M, Holy R, Klotz J, Schaar S, Stoffels G, Galldiks N, Coenen HH, Kaiser HJ, Langen KJ, Eble MJ: Integrated boost IMRT with FET-PET-adapted local dose escalation in glioblastomas. Results of a Prospective Phase II Study. Strahlenther Onkol 2012, 188:334-339.

28. Morganti AG, Balducci M, Salvati M, Esposito V, Romanelli P, Ferro M, Calista F, Digesu C, Macchia G, laniri M, Deodato F, Cilla S, Piermattei A, Valentini V, Cellini N, Cantore GP: A phase I dose-escalation study (ISIDE-BT-1) of accelerated IMRT with temozolomide in patients with glioblastoma. Int J Radiat Oncol Biol Phys 2010, 77:92-97.

29. Watkins JM, Marshall DT, Patel S, Giglio P, Herrin AE, Garrett-Mayer $E_{1}$ Jenrette JM III: High-dose radiotherapy to 78 Gy with or without temozolomide for high grade gliomas. J Neurooncol 2009, 93:343-348. 
30. Terasaki M, Eto T, Nakashima S, Okada Y, Ogo E, Sugita Y, Tokutomi T, Shigemori M: A pilot study of hypofractionated radiation therapy with temozolomide for adults with glioblastoma multiforme. J Neurooncol 2011, 102:247-253

31. Narayana A, Chang J, Thakur S, Huang W, Karimi S, Hou B, Kowalski A, Perera G, Holodny A, Gutin PH: Use of MR spectroscopy and functional imaging in the treatment planning of gliomas. Br J Radiol 2007, 80:347-354.

32. Chang J, Thakur SB, Huang W, Narayana A: Magnetic resonance spectroscopy imaging (MRSI) and brain functional magnetic resonance imaging (FMRI) for radiotherapy treatment planning of glioma. Technol Cancer Res Treat 2008, 7:349-362.

33. Stadlbauer A, Moser E, Gruber S, Nimsky C, Fahlbusch R, Ganslandt O: Integration of biochemical images of a tumor into frameless stereotaxy achieved using a magnetic resonance imaging/magnetic resonance spectroscopy hybrid data Set. J Neurosurg 2004, 101:287-294.

34. Wagner $\mathrm{D}$, Christiansen $\mathrm{H}$, Wolff $\mathrm{H}$, Vorwerk $\mathrm{H}$ : Radiotherapy of malignant gliomas: comparison of volumetric single Arc technique (RapidArc), dynamic intensity-modulated technique and 3D conformal technique. Radiother Oncol 2009, 93:593-596.

35. Douw L, Klein M, Fagel SS, den HJ V, Taphoorn MJ, Aaronson NK, Postma TJ, Vandertop WP, Mooij JJ, Boerman RH, Beute GN, Sluimer JD, Slotman BJ, Reijneveld JC, Heimans JJ: Cognitive and radiological effects of radiotherapy in patients with Low-grade glioma: long-term follow-Up. Lancet Neurol 2009, 8:810-818.

doi:10.1186/1748-717X-8-1

Cite this article as: Ken et al.: Integration method of 3D MR

spectroscopy into treatment planning system for glioblastoma IMRT dose painting with integrated simultaneous boost. Radiation Oncology 2013 8:1.

\section{Submit your next manuscript to BioMed Central and take full advantage of:}

- Convenient online submission

- Thorough peer review

- No space constraints or color figure charges

- Immediate publication on acceptance

- Inclusion in PubMed, CAS, Scopus and Google Scholar

- Research which is freely available for redistribution 